\title{
CONSERVATIVE APPROACH TO PATENT DUCTUS ARTERIOSUS MANAGEMENT IN VERY LOW BIRTH WEIGHT INFANTS
}

\author{
J.B. Letshwiti ${ }^{1}$, K. Pichova ${ }^{2}$, E.M. Dempsey ${ }^{3,4}$, J. Miletin ${ }^{1,2}$ \\ ${ }^{1}$ Coombe Women and Infants University Hospital, Dublin, Ireland, ${ }^{2}$ Institute for the Care of Mother and \\ Child, Prague, Czech Republic, ${ }^{3}$ Cork University Maternity Hospital, ${ }^{4}$ University College Cork, Cork, \\ Ireland
}

Background and aims: The appropriate management of the patent ductus arteriosus (PDA) remains a contentious area. The aim was to evaluate feasibility of conservative management of PDA compared to an early targeted ibuprofen administration and symptomatic medical/surgical treatment.

Methods: Retrospective observational time series cohort study of 3 groups of very low birth weight infants. Infants in the early echo group (EEG) underwent echocardiography evaluation within the first 24 hours of life and received ibuprofen therapy if PDA was larger than $2 \mathrm{~mm}$ in diameter. Infants in symptomatic group (SMG) were echocardiographically evaluated when clinical signs of a PDA were present and treated if significant PDA confirmed. Conservative management group (CMG) included infants with echocardiography on day 7 with present PDA; fluid restriction and increased PEEP was primary mode of treatment.

Results: There were 52 infants in EEG, 52 infants in SMG and 34 infants in CMG. There was no difference in the mean birth weight or mean gestational age between the groups. $48 \%$ of patients in EEG received ibuprofen therapy vs. $62 \%$ in the SMG vs. $15 \%$ in the CMG.19\% infants in the EEG, $21 \%$ patients in the SMG and none in the CMG required PDA ligation. There was no difference in the incidence of death, severe intraventicular haemorrhage or surgical necrotising enterocolitis. There was significantly decreased incidence of chronic lung disease in CMG compared to SMG (17.6\% vs. 50\%; $\mathrm{p}=0.003)$ respectively EEG $(17.6 \%$ vs. $42 \% ; p=0.02)$.

Conclusion: Conservative management of PDA is a feasible option for very low birth weight infants. 